\title{
A literature database on the mating behavior of stylommatophoran land snails and slugs ${ }^{*}$
}

\author{
Angus Davison ${ }^{1}$ and Peter Mordan ${ }^{2}$
}

\author{
${ }^{1}$ Institute of Genetics, School of Biology, University of Nottingham, Queens Medical Centre, Nottingham, NG7 2UH, UK, \\ angus.davison@nottingham.ac.uk \\ ${ }^{2}$ Department of Zoology, The Natural History Museum, London, SW7 5BD, UK, pbm@nhm.ac.uk
}

\begin{abstract}
Stylommatophoran land snails and slugs generally mate by shell-mounting or face-to-face. Although phylogenetic evidence suggests that the mating position has remained more or less constant throughout the evolution of most lineages, other aspects of mating behavior and associated reproductive characters are highly variable. Along with other gastropods, therefore, stylommatophoran land snails and slugs could be particularly useful in trying to understand sex and sex allocation theory in hermaphrodites. It is often difficult, however, to compare mating behavior in different species because the literature is difficult to access or reports have not been formally published. Here we review studies on the mating behavior of snails and slugs, with the additional aim of creating a central access point and database for use as a resource by those interested in stylommatophoran mating behavior. As we maintain the database, updated versions will be made available at http://www.molluscs.org.
\end{abstract}

Key words: love darts, mollusc, sexual conflict, reciprocal mating, simultaneous hermaphrodite

The mating behavior of a wide variety of stylommatophoran land snails has been observed, but the descriptions are often within texts that are not easily accessible, or cannot be searched electronically. Many malacologists have also made their own informal observations of mating behavior, but do not publish them for lack of time, or because they are not perceived to be of sufficient worth on their own. Because there have been no recent reviews of the mating behavior of snails and slugs, we set out to collect as many observations together as possible, both formal and informal. Such an approach has already proved useful in trying to understand how so-called "love" darts evolved (Davison et al. 2005). Differences in mating behavior have also been used to understand the distribution of chiral variation (or asymmetry) among different taxonomic groups (Asami et al. 1998) and the evolution of external sperm exchange (Emberton 1994). The aim of this brief review, therefore, is to create a starting point for a compilation of data on the mating behavior of stylommatophoran snails and slugs.

Although there are some exceptions (e.g., the elongated penes and external fertilization of Limax maximus Linnaeus, 1758), mating in the majority of land snails and slugs can be classified as either face-to-face or shell-mounting (Figure 1; Asami et al. 1998). The vast majority of species are also simultaneous hermaphrodites. In theory, therefore, four different modes of mating are possible because sex is also either simultaneous reciprocal (both individuals are male and female at the same time) or unilateral (each individual has a defined role as male or female during a single round of mating):

Face-to-face, simultaneous reciprocal;

Face-to-face, unilateral;

Shell-mounting, simultaneous reciprocal;

Shell-mounting, unilateral.

When two individuals mate unilaterally, they often switch roles after one round of mating-male becomes female and female becomes male. The frequency with which this occurs is difficult to assess because it requires extended observations, and also the frequency of mate switching depends upon the condition (or desire) of each snail (Koene and Ter Maat 2005). The problem is further complicated because often the most efficient means to make laboratory observations of mating behavior is to isolate individuals for some time before bringing them together. For a variety of possible reasons (e.g., availability of seminal fluid), isolated individuals are more likely to switch mates after one round of mating (Koene and Ter Maat 2005).

Another concern is whether sperm or spermatophore transfer is always reciprocal if mating is reciprocal; from an evolutionary point of view, the reciprocal exchange of sperm is just as important. In some species of Succinea Draparnaud,

\footnotetext{
* From the symposium "Gastropod Mating Systems" presented at the joint meeting of the American Malacological Society and Western Society of Malacologists, held 26-30 June 2005 at Asilomar, Pacific Grove, California.
} 
A.

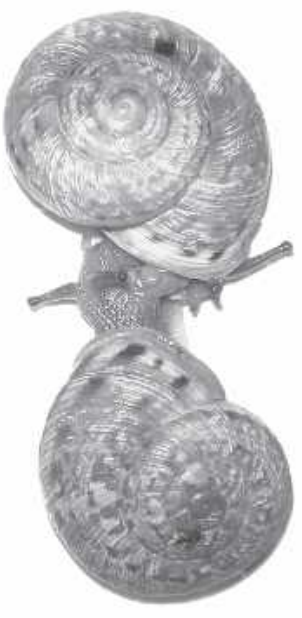

B.

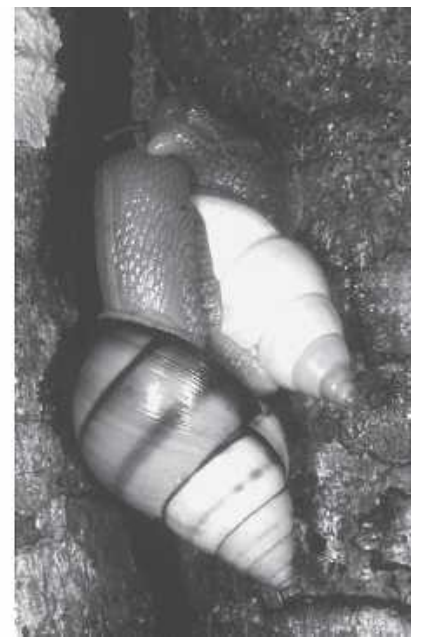

Figure 1. A, Iberus marmoratus Férussac, 1801 (Helicidae) mating face-to-face and simultaneous reciprocally. Horizontal field width approximately $6 \mathrm{~cm}$. Photo by A. Davison, in the laboratory. B, Liguus fasciatus Pilsbry, 1912 (lower snail, acting as male) and Orthalicus floridensis Pilsbry, 1899 (upper snail, acting as female) (Orthalicidae) mating by shell-mounting. Specimens in hardwood hammock in the Redlands of southern Dade County, Florida. Image reproduced with the kind permission of the photographer, Phil Poland, ppoland1@tampabay.rr.com. Vertical field width approx. $7 \mathrm{~cm}$.

1801 that mate reciprocally by shell-mounting, sperm transfer is sometimes unilateral (Jordaens et al. 2005), whereas other species such as Arianta arbustorum Linnaeus, 1758 have a high reciprocity (Baur 1998). In species that develop as males before becoming simultaneous hermaphrodites, such as Lissachatina (Achatina) fulica Bowdich, 1822, sperm transfer may frequently be unilateral (Tomiyama 1993).

We surveyed the formal literature on the mating behavior of land snails and slugs and then classified each species as to whether it mates face-to-face, by shell-mounting, simultaneous reciprocally, or unilaterally (Table 1). We also included informal observations, when available (mating position and, to a lesser extent, reciprocity can be scored from photographs), and tried to identify video recordings of mating behavior (Table 2). One striking, immediately apparent result is that face-to-face mating is exclusively associated with simultaneous reciprocal mating. Snails and slugs in three monophyletic groups mate face-to-face and simultaneous reciprocally: the Helicoidea, Limacoidea, and Philomycidae (Davison et al. 2005).

One other reason to study mating behavior is to understand the evolution of "love" darts. Despite the attention that greets each advance, little is known about the use of darts outside of Cantareus aspersus (Helix aspersa Müller, 1774) (Koene and Chase 1998a, 1998b, Landolfa et al. 2001, Rogers and Chase 2001, Rogers and Chase 2002, Koene and

Schulenberg 2005, Chase 2006), except that there is considerable variation in the timing of the use of the dart, its morphology, and the number used in different species (Ashford 1883, Tompa 1980, Baminger et al. 2000, Koene and Schulenberg 2005). At the extreme of the spectrum, some species of Euhadra Pilsbry, 1890 repeatedly stab a dart ( $\sim 3000$ times) during "foreplay" prior to mating (J. Koene and S. Chiba, personal communication). Opinions also vary over what constitutes a dart. Although some might contend that an "amatorial" organ is a dart, we argue that while darts and amatorial organs may (or may not) have similar functions, they are clearly distinguishable because only the former is a "hard, calcified or chitinous organ that is used to pierce a partner during mating" (Davison et al. 2005).

There have been very few formal observations of dart use. The only photographs that we are aware of showing darts "in use" are of C. aspersus (Koene and Chase 1998a, 1998b, Landolfa et al. 2001, Rogers and Chase 2001, Rogers and Chase 2002, Koene and Schulenberg 2005), Cepaea nemoralis (Davison et al. 2005), and Trichotoxon heynemanni (Schilthuizen 2005). It would therefore be useful if creating a database also stimulated malacologists to record dart use and to publicize their efforts. We have recently used information in the database to show that dart-bearing species are confined to the same three monophyletic groups mentioned above (the Helicoidea, Limacoidea, and Philomycidae) and that they all mate face-to-face and simultaneous reciprocally. However, there is no evidence that the relationship is causal (Davison et al. 2005).

Although there are still some large clades of snails for which there have been few observations of mating behavior, an interesting dichotomy has emerged between invariant mating position and other highly variable reproductive characters. Some species have head warts (Binder 1977, Takeda 1982, Falkner 1993), penial stimulators (Reise 2004), or amatorial organs (Panha 1987), whereas individuals in other species bite off the penis of their partner (Leonard et al. 2002) or entwine their penes before exchanging sperm externally (Quick 1960). Although it has been known that some families tend to have the same shell shape (Cain 1977), the strong and (almost) invariant correlation between mating position and shell shape has mostly been overlooked (Asami et al. 1998, Davison et al. 2005).

Our attention is drawn to the exceptions. In the helicoid group, species of Amphidromus Albers, 1850 have highspired shells but still mate reciprocally, even between chirally-reversed individuals (M. Schilthuizen, personal communication). As they also lack darts, it is tempting to speculate that the shell-shape change and lack of darts are in some way associated, but there is no firm evidence. Clausiild snails are interesting because some species mate unilaterally whereas others mate reciprocally; there is even withinspecies variation (Nordsieck 2005a, 2005b). Finally, Oreohe- 
Table 1. An overview of the literature on the mating behavior of stylommatophoran land snails and slugs. Mating behaviour: FF, face-to-face; I, idiosyncratic; SM, shell-mounting; SR, simultaneous reciprocal; U, unilateral (includes sequential unilateral mating); ?, not known. Shell shape: H, high-spired; L, low-spired; S, slug or semi-slug. Darts: N, dart and art-sac absent; Y, dart or dart-sac present.

\begin{tabular}{|c|c|c|c|c|c|c|}
\hline Family & Genus & \multicolumn{2}{|c|}{ Mating } & $\begin{array}{l}\text { Shell } \\
\text { shape }\end{array}$ & Darts & References \\
\hline \multicolumn{7}{|l|}{ Helicoidea } \\
\hline Bradybaenidae & Euhadra Pilsbry, 1890 & FF & SR & $\mathrm{L}$ & $\mathrm{Y}$ & $\begin{array}{l}\text { Takeda and Tsuruoka 1979, Azuma } \\
\text { 1995, Asami et al. 1998, S. } \\
\text { Chiba, A. Davison, J. Koene, } \\
\text { pers. obs. }\end{array}$ \\
\hline \multirow{4}{*}{ Camaenidae } & Caracolus Montfort, 1810 & $\mathrm{FF}$ & SR & $\mathrm{L}$ & $\mathrm{N}$ & $\begin{array}{l}\text { Howell-Rivero 1950, Webb 1970b, } \\
1974\end{array}$ \\
\hline & Polydontes Montfort, 1810 & $?$ & $?$ & $\mathrm{~L}$ & $\mathrm{~N}$ & Webb 1970b, 1974 \\
\hline & Pleurodonte Fischer von Waldheim, 1807 & $\mathrm{FF}$ & SR & $\mathrm{L}$ & $\mathrm{N}$ & Sánchez Muñoz 2005a \\
\hline & Satsuma A. Adams, 1868 & $\mathrm{FF}$ & SR & $\mathrm{L}$ & $\mathrm{N}$ & Abbott 1989, Azuma 1995 \\
\hline \multirow[t]{6}{*}{ Helicidae } & Cepaea Held, 1837 & $\mathrm{FF}$ & SR & $\mathrm{L}$ & $\mathrm{Y}$ & Beaumont 1988 \\
\hline & Cantareus Risso, 1826 & $\mathrm{FF}$ & SR & $\mathrm{L}$ & $\mathrm{Y}$ & $\begin{array}{l}\text { Giusti and Lepri 1980-1981, } \\
\text { Adamo and Chase 1988, Giusti } \\
\text { and Andreini } 1988\end{array}$ \\
\hline & Theba Risso, 1826 & $\mathrm{FF}$ & SR & $\mathrm{L}$ & $\mathrm{Y}$ & Giusti and Andreini 1988 \\
\hline & Arianta Leach in Turton, 1831 & $\mathrm{FF}$ & SR & $\mathrm{L}$ & $\mathrm{Y}$ & $\begin{array}{l}\text { Hofmann 1923, Locher and Baur } \\
2000\end{array}$ \\
\hline & Helix Linnaeus, 1758 & $\mathrm{FF}$ & SR & $\mathrm{L}$ & $\mathrm{Y}$ & $\begin{array}{l}\text { Meisenheimer 1907, Jeppesen 1976, } \\
\text { Lind 1976, Giusti and Lepri } \\
\text { 1980-1981, Chung } 1987\end{array}$ \\
\hline & Tacheocampylaea Pfeiffer, 1877 & $\mathrm{FF}$ & SR & $\mathrm{L}$ & $\mathrm{Y}$ & Giusti and Lepri 1980-1981 \\
\hline \multirow{3}{*}{ Helminthoglyptidae } & Monadenia Pilsbry, 1895 & $\mathrm{FF}$ & SR & $\mathrm{L}$ & $\mathrm{Y}$ & Webb $1952 b$ \\
\hline & Sonorella Pilsbry, 1900 & $\mathrm{FF}$ & SR & $\mathrm{L}$ & & Webb 1980a, 1990 \\
\hline & Polymita Beck, 1837 & $\mathrm{FF}$ & SR & $\mathrm{L}$ & $\mathrm{Y}$ & Moreno 1950, Tur et al. 2002 \\
\hline \multirow[t]{3}{*}{ Hygromiidae } & Cochlicella Férussac, 1820 & $\mathrm{FF}$ & SR & $\mathrm{H}$ & $\mathrm{Y}$ & $\begin{array}{l}\text { Schileyko and Menkhorst 1997, } \\
\text { Asami et al. } 1998\end{array}$ \\
\hline & Monacha Fitzinger, 1833 & FF & SR & $\mathrm{L}$ & $\mathrm{Y}$ & Storey 2005 \\
\hline & Halolimnohelix Germain, 1913 & $\mathrm{FF}$ & SR & $\mathrm{L}$ & $\mathrm{Y}$ & Block 1968a \\
\hline \multirow[t]{6}{*}{ Polygyridae $^{2}$} & Allogona Pilsbry, 1939 & $\mathrm{FF}$ & SR & $\mathrm{L}$ & $\mathrm{N}$ & $\begin{array}{l}\text { Webb 1948a, Emberton } 1994 \text {, } \\
\text { Atkinson } 2005\end{array}$ \\
\hline & Ashmunella Pilsbry \& Cockerell, 1899 & $\mathrm{FF}$ & SR & $\mathrm{L}$ & $\mathrm{N}$ & Webb 1954a, Emberton 1994 \\
\hline & Cryptomastix Pilsbry, 1939 & $\mathrm{FF}$ & SR & $\mathrm{L}$ & $\mathrm{N}$ & Webb 1970c, Emberton 1994 \\
\hline & Mesodon Rafinesque in Férussac, 1821 & $\mathrm{FF}^{1}$ & SR & $\mathrm{L}$ & $\mathrm{N}$ & Webb 1954b, Emberton 1991 \\
\hline & Neohelix Ihering, 1892 & $\mathrm{FF}$ & SR & $\mathrm{L}$ & $\mathrm{N}$ & Webb 1952d, Emberton 1994 \\
\hline & Polygyra Say, 1818 & $\mathrm{FF}^{1}$ & SR & $\mathrm{L}$ & $\mathrm{N}$ & $\begin{array}{l}\text { Archer 1933, Emberton 1994, } \\
\text { Webb 1994a, 1994b }\end{array}$ \\
\hline
\end{tabular}


Table 1. Continued

\begin{tabular}{|c|c|c|c|c|c|c|}
\hline \multirow[t]{4}{*}{ Family } & \multirow{2}{*}{$\begin{array}{l}\text { Genus } \\
\text { Stenotrema Rafinesque, } 1819\end{array}$} & \multicolumn{2}{|c|}{ Mating } & \multirow{2}{*}{$\begin{array}{l}\begin{array}{l}\text { Shell } \\
\text { shape }\end{array} \\
\text { L }\end{array}$} & \multirow{2}{*}{$\begin{array}{l}\text { Darts } \\
\mathrm{N}\end{array}$} & \multirow{2}{*}{$\begin{array}{l}\text { References } \\
\text { Webb 1947, 1948b, Emberton } 1994\end{array}$} \\
\hline & & $\mathrm{FF}^{1}$ & SR & & & \\
\hline & Triodopsis Rafinesque, 1819 & $\mathrm{FF}$ & SR & $\mathrm{L}$ & $\mathrm{N}$ & Webb 1948a, 1959, Emberton 1994 \\
\hline & Vespericola Pilsbry, 1939 & $\mathrm{FF}$ & SR & $\mathrm{L}$ & $\mathrm{N}$ & Webb 1970a, Emberton 1994 \\
\hline \multicolumn{7}{|l|}{ Limacoidea } \\
\hline \multirow{2}{*}{ Arionidae } & Geomalacus Allman, 1842 & $\mathrm{FF}$ & $?$ & $\mathrm{~S}$ & $\mathrm{~N}$ & Platts and Speight 1988 \\
\hline & Ariolimax Mörch, 1859 & $\mathrm{FF}$ & $\mathrm{SR} / \mathrm{U}$ & $\mathrm{S}$ & $\mathrm{N}$ & Leonard et al. 2002 \\
\hline \multirow[t]{2}{*}{ Ariophantidae } & Ariophanta Desmoulins, 1829 & $\mathrm{FF}$ & SR & $\mathrm{L}$ & $\mathrm{Y}$ & Dasen 1933 \\
\hline & Hemiplecta Albers, 1850 & $\mathrm{FF}$ & SR & $\mathrm{L}$ & $\mathrm{N}$ & S. Panha, pers. comm. \\
\hline Limacidae & Limacus Lehmann, 1864 & FF & SR & $\mathrm{S}$ & $\mathrm{N}$ & Barker 1999 \\
\hline Milacidae & Tandonia (Milax) Lesson \& Pollonera, 1882 & $\mathrm{FF}$ & SR & $\mathrm{S}$ & $\mathrm{N}$ & Quick 1960 \\
\hline Trochomorphidae & Bertia Ancey, 1887 & $\mathrm{FF}$ & SR? & $\mathrm{L}$ & $\mathrm{N}$ & Menno Schilthuizen, pers. comm. \\
\hline \multirow[t]{4}{*}{ Urocyclidae } & Trichotoxon Simroth, 1889 & $\mathrm{FF}$ & SR & $\mathrm{S}$ & $\mathrm{Y}$ & Bernard Verdcourt, pers. comm. \\
\hline & Gymnarion Pilsbry, 1919 & $\mathrm{FF}$ & SR & $\mathrm{L}$ & $\mathrm{N}$ & Binder 1977 \\
\hline & Sheldonia Ancey, 1887 & $\mathrm{FF}$ & SR & $\mathrm{L}$ & $\mathrm{N}$ & Herbert and Kilburn 2004 \\
\hline & Elisolimax Cockerell, 1893 & $\mathrm{FF}$ & SR & $\mathrm{S}$ & $\mathrm{N}$ & Herbert and Kilburn 2004 \\
\hline \multirow[t]{2}{*}{ Vitrinidae } & Semilimax Agassiz, 1845 & FF & SR & $\mathrm{S}$ & $\mathrm{Y}$ & Künkel 1933 \\
\hline & Vitrinobrachium Kunkel, 1929 & FF & SR & $\mathrm{S}$ & $\mathrm{N}$ & Künkel 1933 \\
\hline Zonitidae & Mesomphix Rafinesque, 1819 & $\mathrm{FF}$ & SR & $\mathrm{L}$ & $\mathrm{N}$ & Webb $1952 c$ \\
\hline Chondrinidae & Solatopupa Pilsbry, 1917 & $?$ & $\mathrm{U}$ & $\mathrm{H}$ & $\mathrm{N}$ & Boato and Rasotto 1987 \\
\hline \multirow[t]{4}{*}{ Clausiliidae } & Albinaria Vest, 1867 & $\mathrm{SM}$ & SR & $\mathrm{H}$ & $\mathrm{N}$ & $\begin{array}{l}\text { Schilthuizen and Lombaerts } 1995 \text {, } \\
\text { Menno, Schilthuizen, pers. comm. }\end{array}$ \\
\hline & Euphaedusa O. Boettger, 1877 & $\mathrm{SM}$ & $\mathrm{U}$ & $\mathrm{H}$ & $\mathrm{N}$ & Asami et al. 1998 \\
\hline & Luchuphaedusa Pilsbry, 1901 & SM & $\mathrm{U}$ & $\mathrm{H}$ & $\mathrm{N}$ & Asami et al. 1998 \\
\hline & Stereophaedusa O. Boettger, 1877 & SM & $\mathrm{U}$ & $\mathrm{H}$ & $\mathrm{N}$ & Asami et al. 1998 \\
\hline \multirow[t]{5}{*}{ (Alopilinae) } & Agathylla H. \& A. Adams, 1855 & SM & SR & $\mathrm{H}$ & $\mathrm{N}$ & Nordsieck 1969, 2005a, 2005b \\
\hline & Cochlodina Férussac, 1821 & SM & $\mathrm{U}$ & $\mathrm{H}$ & $\mathrm{N}$ & Nordsieck 2005a, 2005b \\
\hline & Delima Hartmann, 1842 & SM & SR & $\mathrm{H}$ & $\mathrm{N}$ & Nordsieck 1969, 2005a, 2005b \\
\hline & Herilla H. \& A. Adams, 1855 & SM & SR & $\mathrm{H}$ & $\mathrm{N}$ & Nordsieck 2005a, 2005b \\
\hline & Medora H. \& A. Adams, 1855 & SM & SR & $\mathrm{H}$ & $\mathrm{N}$ & Nordsieck 2005a, 2005b \\
\hline (Baleinae) & Balea Gray, 1824 & SM & $\mathrm{U}$ & $\mathrm{H}$ & $\mathrm{N}$ & Nordsieck 2005a, 2005b \\
\hline \multirow[t]{5}{*}{ (Clausiliinae) } & Laciniaria Hartmann, 1842 & SM & $\mathrm{U}$ & $\mathrm{H}$ & $\mathrm{N}$ & Nordsieck 2005a, 2005b \\
\hline & Macrogastra Hartmann, 1841 & $\mathrm{SM}$ & $\mathrm{U}$ & $\mathrm{H}$ & $\mathrm{N}$ & Nordsieck 2005a, 2005b \\
\hline & Clausilia Draparnaud, 1805 & SM & $\mathrm{U}$ & $\mathrm{H}$ & $\mathrm{N}$ & Nordsieck 2005a, 2005b \\
\hline & Ruthenica Lindholm, 1924 & SM & $\mathrm{U}$ & $\mathrm{H}$ & $\mathrm{N}$ & Nordsieck 2005a, 2005b \\
\hline & Neostyriaca A. Wagner, 1920 & SM & $\mathrm{U}$ & $\mathrm{H}$ & $\mathrm{N}$ & Nordsieck 2005a, 2005b \\
\hline
\end{tabular}


Table 1. Continued

\begin{tabular}{|c|c|c|c|c|c|c|}
\hline \multirow{2}{*}{$\frac{\text { Family }}{\text { Discidae }}$} & \multirow{2}{*}{$\begin{array}{l}\text { Genus } \\
\text { Anguispira Morse, } 1864\end{array}$} & \multicolumn{2}{|c|}{ Mating } & \multirow{2}{*}{$\begin{array}{l}\begin{array}{l}\text { Shell } \\
\text { shape }\end{array} \\
\text { L }\end{array}$} & \multirow{2}{*}{$\begin{array}{l}\text { Darts } \\
\mathrm{N}\end{array}$} & \multirow{2}{*}{$\begin{array}{l}\text { References } \\
\text { Webb 1968b }\end{array}$} \\
\hline & & $\mathrm{SM}^{1}$ & SR & & & \\
\hline Haplotrematidae & Haplotrema Ancey, 1881 & SM & $\mathrm{U}$ & $\mathrm{L}$ & $\mathrm{N}$ & Webb 1943 \\
\hline Oreohelicidae & Oreohelix Pilsbry, 1904 & $\mathrm{SM}$ & $\mathrm{U}$ & $\mathrm{L}$ & $\mathrm{N}$ & Webb 1951 \\
\hline Philomycidae & Philomycus Rafinesque, 1820 & FF & SR & $\mathrm{S}$ & $\mathrm{Y}$ & Webb 1968a \\
\hline Rhytididae & Paryphanta Albers, 1850 & $\mathrm{SM}$ & $\mathrm{U}$ & $\mathrm{L}$ & $\mathrm{N}$ & Stringer et al. 2003 \\
\hline Spiraxidae & Euglandina Fischer \& Crosse, 1870 & $\mathrm{SM}$ & $\mathrm{U}$ & $\mathrm{H}$ & $\mathrm{N}$ & Cook 1985 \\
\hline Strophocheilidae & Strophocheilus Spix, 1827 & $\mathrm{SM}$ & $\mathrm{U}$ & $\mathrm{H}$ & $\mathrm{N}$ & Wiswell and Browning 1967 \\
\hline Succineidae & Catinella Pease, 1870 & $\mathrm{SM}^{1}$ & SR & $\mathrm{H}$ & $\mathrm{N}$ & Webb $1977 \mathrm{a}$ \\
\hline Valloniidae & Vallonia Risso, 1826 & FF & SR? & $\mathrm{L}$ & $\mathrm{N}$ & Barker 1999 \\
\hline Vertiginidae & Vertigo Müller, 1774 & $\mathrm{SM}$ & $\mathrm{U} ?$ & $\mathrm{H}$ & $\mathrm{N}$ & Barker 1999 \\
\hline
\end{tabular}

${ }^{1}$ External sperm exchange; sperm is deposited on the male's everted penis without intromission (see Emberton 1994)

${ }^{2}$ See Emberton (1994) for details of mating in other Polygyrid species (mostly papers by Webb).

Table 2. Species for which there are videos showing mating behavior.

\begin{tabular}{|c|c|c|}
\hline Taxon & $\begin{array}{l}\text { Mating } \\
\text { behavior }\end{array}$ & Film-maker \\
\hline Ariolimax dolichophallus Mead, 1943 & Face-to-face & $\begin{array}{l}\text { Brooke Miller, UC Santa Cruz (miller@biology.ucsc.edu) } \\
\text { http://bio.research.ucsc.edu/grad/weaver/Pages/project.html }\end{array}$ \\
\hline Cantareus aspersus Müller, 1774 & Face-to-face & $\begin{array}{l}\text { Joris Koene, Vrije Universiteit (joris.koene@falw.vu.nl) } \\
\text { Ronald Chase, McGill University (ronald.chase@mcgill.ca) }\end{array}$ \\
\hline Deroceras sp. & Face-to-face & $\begin{array}{l}\text { Heike Reise, Staatliches Museum fur Naturkunde Gorlitz } \\
\text { (Heike.Reise@smng.smwk.sachsen.de } \\
\text { http://www.malacsoc.org.uk/Malacological\%20Bulletin/BULL43/king2.htm\#dive }\end{array}$ \\
\hline Euhadra sandai Kobelt, 1879 & Face-to-face & $\begin{array}{l}\text { Nishi Hirotaka (movie archives of animal behaviour) } \\
\text { http://zoo2.zool.kyoto-u.ac.jp/ethol/ }\end{array}$ \\
\hline Satsuma amanoi Kuroda, 1960 & Face-to-face & $\begin{array}{l}\text { Nishi Hirotaka (movie archives of animal behaviour) } \\
\text { http://zoo2.zool.kyoto-u.ac.jp/ethol/ }\end{array}$ \\
\hline Mastus pupa Linnaeus, 1758 & Shell-mounting & Aris Paramakelis, University of Crete (parmakel@nhmc.uoc.gr) \\
\hline
\end{tabular}

lix Pilsbry, 1904 is another intriguing genus because it mates by shell-mounting (Webb 1951). Although its phylogeny is unclear (Wade et al. 2006), we predict that it will either fall within the helicoid group or be basal to it.

We expect that observations of mating behavior will grow over the coming years and so will continue to update the database of mating behavior (updated versions at http:// www.molluscs.org). We welcome any data that we have inadvertently excluded, including single photographs.

\section{ACKNOWLEDGEMENTS}

We would like to thank Glenn Webb for making so many observations over so many years. This paper would 
not have been possible without his contribution. Thanks also to comments received from two anonymous referees.

\section{LITERATURE CITED}

Abbott, R. T. 1989. Compendium of landshells. American Malacologists, Inc., Melbourne, Florida.

Adamo, S. A. and R. Chase. 1988. Courtship and copulation in the terrestrial snail Helix aspersa. Canadian Journal of Zoology 66: 1446-1453.

Adams, L. E. 1910. Observations on the pairing of Arion ater (L.). Journal of Conchology 13: 116-119.

Archer, A. F. 1933. A study of Polygyra inflecta (Say). Occasional Paper of the Museum of Zoology of the University of Michigan. 8 pp.

Asami, T., R. H. Cowie, and K. Ohbayashi. 1998. Evolution of mirror images by sexually asymmetric mating behavior in hermaphroditic snails. American Naturalist 152: 225-236.

Ashford, C. 1883. The darts of British Helicidae. Journal of Conchology 4: 69-320.

Atkinson, J. W. 2005. A pair of Allogona profunda mating in the laboratory. Available at http://www.msu.edu/ atkinso9/ gallery.htm 27 July 2006.

Azuma, M. 1995. Coloured illustrations of the land snails of Japan. Hoikusya, Osaka. [In Japanese.]

Baminger, H., R. Locher, and B. Baur. 2000. Incidence of dart shooting, sperm delivery, and sperm storage in natural populations of the simultaneously hermaphroditic land snail Arianta arbustorum. Canadian Journal of Zoology 78: 1767-1774.

Barker, G. M. 1999. Naturalised Terrestrial Stylommatophora. Landcare Research, Lincoln, Canterbury.

Baur, B. 1998. Sperm competition in molluscs. In: T. Birkhead and A. P. Møller, eds., Sperm competition and sexual selection. Academic Press, London. Pp. 255-305.

Beaumont, M. A. 1988. A study of reproductive interference between closely related species. Ph.D Dissertation. University of Nottingham, UK.

Binder, E. 1977. La pariade chez le genre Gymnarion Pilsbry 1919; role de l'organe frontal. Archiv für Molluskenkunde 107: 249255.

Block, M. R. 1968a. Some observations on Haloliomnohelix iredalei Connolly. Journal of Conchology 26: 219-224.

Block, M. R. 1968b. The anatomy of Zebrinops ventricosa Connolly, with notes on the living animal. Revue de Zoologie et de Botanique Africaine 78: 325-331.

Boato, A. and M. B. Rasotto. 1987. Functional protandry and seasonal reproductive cycle in Solatopupa similis Bruguière (Pulmonata, Chondrinidae). Bolletino di Zoologia 54: 119-125.

Cain, A. J. 1977. Variation in spire index of some coiled gastropod shells and its evolutionary significance. Philosophical Transactions of the Royal Society of London B Biological Sciences 277: 377-428.

Chase, L. M. 1952. The aerial mating of the great slug. Discovery 13: 356-359.
Chase, R. 2007. The function of dart shooting in certain helicid snails. American Malacological Bulletin 23: 183-189.

Chung D. J. D. 1987. Courtship and dart shooting behavior of the land snail Helix aspersa. Veliger 30: 24-39.

Cook, A. 1985. The courtship of Euglandina rosea (Férussac). Journal of Molluscan Studies 51: 211-214.

Dasen, D. D. 1933. Structure and function of the reproductive system in Ariophanta. Proceedings of the Zoological Society of London 1933: 97-123.

Davidson, T. 1965. Tree snails. Gems of the Everglades. National Geographic Magazine 127: 375.

Davis, S. M. 1977. The Arion hortensis complex, with notes on Arion intermedius Normand (Pulmonata: Arionidae). Journal of Conchology 29: 173-187.

Davison, A., C. M. Wade, P. B. Mordan, and S. Chiba. 2005. Sex and darts in slugs and snails (Mollusca: Gastropoda: Stylommatophora). Journal of Zoology 267: 329-338.

Emberton, K. C. 1991. The genitalic, allozymic and conchological evolution of the tribe Mesodontini (Pulmonata: Stylommatophora: Polygyridae). Malacologia 33: 71-178.

Emberton, K. C. 1994. Polygyrid land snail phylogeny-external sperm exchange, early North-American biogeography, iterative shell evolution. Biological Journal of the Linnean Society 52: 241-271.

Falkner, G. 1993. Lockspiel und lockstoffdrüsen bei Hygromiiden und Heliciden (Gastropoda: Stylommatophora). Heldia 2: 1520.

Giusti, F. and S. Andreini. 1988. Morphological and ethological aspects of mating in two species of the family Helicidae (Gastropoda Pulmonata): Theba pisana (Müller) and Helix aspersa Born. Monitore Zoologico Italiano Monografia, New series 22: 331-363.

Giusti, F. and A. Lepri. 1980-1981. Aspetti morfologici ed etologici dell'accoppiamento in alcune specie della famiglia Helicidae (Gastropoda, Pulmonata). Lavori della Società Malacologica Italiana 17-18: 11-71.

Hecker, U. 1965. Zur Kenntnis der mitteleuropäischen Bernsteischenecken. Archiv für Molluskenkunde 94: 1-45.

Herbert, D., and D. Kilburn. 2004. Field guide to the land snails and slugs of eastern South Africa. Natal Museum, South Africa.

Hofmann, E. 1923. Ueber den Begattungsvorgang von Arianta arbustorum (L.). Jenaische Zeitschrift für Naturwissenschaften 59: 363-400.

Howell-Rivero, L. 1946. Nota sobre la cópula de Zachrysia guanensis y Veronicella tenax. Revista de la Sociedad Malacólogica "Carlos de la Torre y Huerta" 4: 75-84.

Howell-Rivero, L. 1950. Reproducción de Caracolus sagemon Beck. Revista de la Sociedad Malacólogica "Carlos de la Torre y Huerta" 7: 45-50.

Jackiewicz, M. 1980. Some observations on biology of reproduction of Succinea Draparnaud (Gastropoda, Pulmonata). Annales Zoologici 35: 65-73.

Jeppesen, L. L. 1976. The control of mating behaviour in Helix pomatia L. (Gastropoda: Pulmonata). Animal Behaviour 24: 275-290.

Jordaens, K., J. Pinceel and T. Backeljau. 2005. Mate choice in the 
hermaphroditic land snail Succinea putris (Stylommatophora: Succineidae). Animal Behaviour 70: 329-337.

Koene, J. M. and R. Chase. 1998a. Changes in the reproductive system of the snail Helix aspersa caused by mucus from the love dart. Journal of Experimental Biology 201: 2313-2319.

Koene, J. M. and R. Chase. 1998b. The love dart of Helix aspersa MưlLER is not a gift of calcium. Journal of Molluscan Studies 64: 75-80.

Koene, J. M. and H. Schulenberg. 2005. Shooting darts: coevolution and counter-adaptation in hermaphroditic snails. BMC Evolutionary Biology 5: 25.

Koene, J. M. and A. Ter Maat. 2005. Sex role alternation in the simultaneously hermaphroditic pond snail Lymnaea stagnalis is determined by the availability of seminal fluid. Animal Behaviour 69: 845-850.

Künkel, K. 1933. Vergleichende experimentelle studie über Vitrina elongata Draparnaud und Vitrina brevis Férussac. Zoologische Jahrbucher 52: 399-432.

Landolfa, M. A., D. M. Green, and R. Chase. 2001. Dart shooting influences paternal reproductive success in the snail Helix aspersa (Pulmonata, StYlommatophora). Behavioral Ecology 12: 773-777.

Langlois, T. 1965. The conjugal behavior of the introduced European giant garden slug Limax maximus as observed on S. Bass Island, Lake Erie. Ohio Journal of Science 65: 208-304.

Leonard, J. L., J. S. Pearse, and A. B. Bryant Harper. 2002. Comparative reproductive biology of Ariolimax californicus and A. dolichophallus (Gastropoda: Stylommatophora). Invertebrate Reproduction and Development 41: 83-93.

Lind, H. 1976. Causal functional organization of the mating behaviour sequence in Helix pomatia (Pulmonata, Gastropoda). Behavior 59: 162-201.

Lipton, C. S. and J. Murray. 1979. Courtship of land snails of the genus Partula. Malacologia 19: 129-146.

Locher, R. and B. Baur. 2000. Sperm delivery and egg production of the simultaneously hermaphroditic land snail Arianta arbustorum exposed to an increased sperm competition risk. Invertebrate Reproduction and Development 38: 53-60.

Meisenheimer, J. 1907. Biologie, Morpholgie und Physiologie des Begattungvorganges und der Eiablage von Helix pomatia. Zoologische Jahrbücher Abteilung für Systematik und Oekologie 25: 461-502.

Moreno, A. 1950. Estudio anatómico del género Polymita Beck. Memorias de la Sociedad Cubana de Historia Natural "Felipe Poey" 20: 21-35.

Nordsieck, H. 1969. Zur Anatomie und Systematik der Clausilien, VI. Genitalsystem und Systematik der Clausiliidae, besonders der Unterfamilie Alopiinae. Archiv für Molluskenkunde 99: 247-265.

Nordsieck, H. 2005a. Mating biology of Clausiliidae (Gastropoda, Stylommatophora). http://www.clausilia.de, accessed 18 October 2006.

Nordsieck, H. 2005b. Mating biology of Clausiliidae (Gastropoda: Stylommatophora). Mitteilungen der Deutschen Malakozoologischen Gesellschaft 73/74: 29-34.

Panha, S. 1987. Histochemical and ultrastructural studies on the amatorial organ of Hemiplecta distincta (Pfeiffer) (Pulmonata: Ariophantidae). Venus 46: 109-115.

Paramakelis, A. and M. Mylonas. 2002. Aspects of the reproduction and activity of two sympatric Mastus (Beck, 1837) species in Crete (Gastropoda: Pulmonata: Buliminidae). Journal of Molluscan Studies 68: 225-233.

Pilsbry, H. A. 1946. Land mollusca of North America (North of Mexico). The Academy of Natural Sciences of Philadelphia, Philadelphia, Pennsylvania.

Platts, E. A. and M. C. D. Speight. 1988. The taxonomy and distribution of the Kerry slug Geomalacus maculosus Allman, 1843 (Mollusca: Arionidae) with a discussion of its status as a threatened species. The Irish Naturalists' Journal 22: 417-430.

Plummer, J. M. 1975. Observations on the reproduction, growth and longevity of a laboratory colony of Archachatina (Calachatina) marginata (Swainson) subspecies ovum. Journal of Molluscan Studies 41: 395-413.

Poland, P. 2005. Mating time in the hammock (Liguus fasciatus lossmanicus Pilsbry, 1912). Available at: http://www.jaxshells .org/liguusf.htm 27 July 2006.

Quick, H. E. 1946. The mating process in Arion hortensis Férussac and in Arion subfuscus Draparnaud. Journal of Conchology 22: 178-182.

Quick, H. E. 1960. British slugs (Pulmonata: Testacellidae, Arionidae Limacidae). Bulletin of the British Museum of Natural History and Zoology 6: 105-226.

Rabaneda-Bueno, R., G. Moreno-Rueda, F. A. Rulz-Avilés, and R. Márquez-Ferrando. 2004. Trio mating formation during copula in the hermaphrodite land snail Iberus gualtierianus L. (Gastropoda: Helicidae). Available at: http://www.malacsoc .org.uk/Malacological\%20Bulletin/BULL43/king2.htm\#trio 27 July 2006

Reise, H. 1995. Mating behavior of Deroceras rodnae Grossu and Lupu, 1965 and Deroceras praecox Wiktor, 1966 (Pulmonata, Agriolimacidae). Journal of Molluscan Studies 61: 325-330.

Reise, H. 2004. Diversity of mating behaviour within Deroceras. Malacological Society Bulletin 43: 11.

Rieper, H. 1912. Studien an Succinea. Annales de la Societe Royale Zoologique et Malacologique de Belgique 27: 125-191.

Rodriguez, A. S. and B. J. Gomez. 1999. Copulatory process in Oxychilus (Drouetia) atlanticus (Morelet \& Drouët, 1857) (Pulmonata: Zonitidae). Invertebrate Reproduction and Development 36: 1-3.

Rogers, D. W. and R. Chase. 2001. Dart receipt promotes sperm storage in the garden snail Helix aspersa. Behavioral Ecology and Sociobiology 50: 122-127.

Rogers, D. W. and R. Chase. 2002. Determinants of paternity in the garden snail Helix aspersa. Behavioral Ecology and Sociobiology 52: 289-295.

Sánchez Muñoz, A. J. 2005a. Mating pair of camaenid snails, Pleurodonte excellens. Santo Domingo, southern Dominican Republic, Hispaniola. Available at: http://www.kingsnake.com/ westindian/pleurodonteexcellens1.JPG 27 July 2006.

Sánchez Muñoz, A. J. 2005b. Camaenid snails, probably Zachrysia provisoria, during mating. Barrenspot, central Saint Croix, United States Virgin Islands. Available at: http://www 
.kingsnake.com/westindian/zachrysiaprovisorial.JPG 27 July 2006.

Schileyko, A. A. and H. P. M. G. Menkhorst. 1997. Composition and phylogenetic relations of the Cochlicellidae (Gastropoda, Pulmonata). Ruthenica 7: 51-60.

Schilthuizen, M. 2005. The darting game in snails and slugs. Trends in Ecology and Evolution 20: 581-584.

Schilthuizen, M. and A. Davison. 2005. The convoluted evolution of snail chirality. Naturwissenschaften 92: 504-515.

Schilthuizen, M., and M. Lombaerts. 1995. Life on the edge-a hybrid zone in Albinaria hippolyti (Gastropoda, Clausiliidae) from Crete. Biological Journal of the Linnean Society 54: 111138.

Storey, M. 2005. BioImages—virtual field-guide. http:www.bioimages .org.uk, accessed 18 October 2006.

Stringer, I. A. N., S. M. Bassett, M. J. McLean, J. McCartney, and G. R. Parrish. 2003. Biology and conservation of the rare New Zealand land snail Paryphanta busbyi watti (Mollusca, Pulmonata). Invertebrate Biology 122: 241-251.

Takeda, N. 1982. Notes on the fine structure of the head-wart in some terrestrial snails. The Veliger 24: 328-330.

Takeda, N., and H. Tsuruoka. 1979. A sex pheremone secreting gland in the terrestrial snail, Euhadra peliomphala. Journal of Experimental Zoology 207: 17-26.

Tomiyama, K. 1993. Growth and maturation pattern of African giant snail, Achatina fulica (Férussac) (Stylommatophora; Achatinidae) in Ogasawara Islands. Venus 51: 85-98.

Tomiyama, K. 1994. Courtship behaviour of the giant African land snail Achatina fulica (Férussac) (Stylomatophora: Achatinidae) in the field. Journal of Molluscan Studies 60: 47-54.

Tomiyama, K. 1996. Mate-choice criteria in a protandrous simultaneously hermaphroditic land snail Achatina fulica (Ferussac) (Stylommatophora: Achatinidae). Journal of Molluscan Studies 62: 101-111.

Tompa, A. S. 1980. The ultrastructure and mineralogy of the dart from Philomycus carolinianus (Pulmonata: Gastropoda) with a brief survey of the occurrence of darts in land snails. The Veliger 23: 35-42.

Tur, B. R., A. F. Velázquez and Y. O. Cabrera. 2002. Conducta de apareamiento y aspectos de la relación del sistema reproductor en Polymita muscarum Lea 1834 (Gastropoda: Pulmonata). Revista Biologia 14: 160-166.

van der Laan, K. L. 1971. The population ecology of the terrestrial snail, Helminthoglypta arrosa (Pulmonata: Helicidae). Ph.D. dissertation. University of California Berkeley, California.

van der Laan, K. L. 1980. Terrestrial pulmonate reproduction: Seasonal and annual variation and environmental factors in $\mathrm{Hel}$ minthoglypta arrosa (Binney) (Pulmonata: Helicidae). The Veliger 23: 48-54.

Villalobos, C., J. Monge-Nájera, Z. Barrientos, and J. Franco. 1995. Life cycle and field abundance of the snail Succinea costaricana (Stylommatohphora: Succineidae), a tropical agricultural pest. Revista de Biologia Tropical 43: 181-188.

Wade, C. M., P. B. Mordan, and F. Naggs. 2006. Evolutionary relationships among the Pulmonate land snails and slugs (Pul- monata, Stylommatophora). Biological Journal of the Linnean Society 87: 593-610.

Webb, G. R. 1942. Comparative observations of the mating habits of three California landsnails. Bulletin of the Southern California Academy of Science 41: 102-108.

Webb, G. R. 1943. The mating of the landsnail Haplotrema concavum (Say). American Midland Naturalist 30: 341-345.

Webb, G. R. 1947. Studies of the sex-organs of mating polygyrid landsnails. Ill. Transactions of the Illinois State Academy of Science 40: 218-227.

Webb, G. R. 1948a. Comparative observations on the mating of certain Triodopsinae. Nautilus 61: 97-103.

Webb, G. R. 1948b. The mating of Stenotrema fraternum (Say). Nautilus 62: 8-12.

Webb, G. R. 1948c. Notes on the mating of some zonitoides (Ventridens) species of land snails. American Midland Naturalist 40: 453-461.

Webb, G. R. 1951. Sexological notes on the landsnail Oreohelix. Natural History Miscellanea 78: 1-5.

Webb, G. R. 1952a. Pulmonata, Helmithoglyptidae: Sexological data on the land-snails, Cepolis maynardi \& Helminthoglypta traski and their evolutionary significance. Gastropodia 1: 3-5.

Webb, G. R. 1952b. Pulmonata, Xanthonycidae: Comparative sexological studies of the North American land-snail, Monadenia fidelis (Gray) - a seeming ally of Mexican helicoids. Gastropodia 1: 1-3.

Webb, G. R. 1952c. Sexological notes on Mesomphix cupreus and M. subplanus. Transactions of the American Microscopical Society 71: 408-410.

Webb, G. R. 1952d. A sexological revision of some triodopsin landsnails, Xolotrema, Neohelix, and Wilcoxorbis. Gastropodia 1: 7-8.

Webb, G. R. 1954a. The life-history and sexual anatomy data on Ashmunella with a revision of the triodopsin snails. Gastropodia 1: 11-18.

Webb, G. R. 1954b. Pulmonata, Polygyridae, Polygyrinae: The sexology and taxonomy of seven species of land-snails of the genus Mesodon. Gastropodia 1: 19-20.

Webb, G. R. 1959. Pulmonata, Polygyridae: Notes on the sexology of Triodopsis, a new sub-genus, Haroldorbis, and a new section, Shelfordorbis. Gastropodia 1: 23-25.

Webb, G. R. 1965. On the sexology of Trilobopsis loricata sonomaen sis (Hemphill). Gastropodia 1: 58-60.

Webb, G. R. 1968a. Observations on the sexology of Philomycus carolinianus Bosc. Gastropodia 1: 62.

Webb, G. R. 1968b. Observations on the sexology of the endodontid land-snail, Anguispira alternata (Say). Gastropodia 1: 66-67.

Webb, G. R. 1970a. Observations on the sexology of Vespericola columbiana (Lea) from Olympic Peninsula, Washington. Gastropodia 1: 66-67.

Webb, G. R. 1970b. Pulmonata, Camaenidae: Comparative sexology and genital development of Caracolus caracolla (L.), C. marginella (Gmelin), and Polydontes lima (Ferrussac). Gastropodia 1: 79-84. 
Webb, G. R. 1970c. Sexological notes on Cryptomastix mullani (Bland \& Cooper). Gastropodia 1: 74-75.

Webb, G. R. 1974. Pulmonata, Camaenidae: Comparative sexology and genital development of Caracolus caracolla (L.), C. marginella (Gmelin), and Polydontes lima (Ferrussac). Gastropodia 1: 90-95.

Webb, G. R. 1977a. The comparative sexology of several Succineidae. Gastropodia 1: 105-107.

Webb, G. R. 1977b. On the sexology of Catinella (Mediappendix) avara (Say) or C. (M.) vermeta (Say). Gastropodia 1: 100-102.

Webb, G. R. 1977c. Some sexologic observations on Oxyloma retusa (Lea). Gastropodia 1: 102-104.

Webb, G. R. 1980a. Notes on the sexology of Sonorella (Masculus) virilis Pilsbry, Pulmonata, Xanthonysidae. Gastropodia 2: 7-8.

Webb, G. R. 1980b. The sexology of a Texan Humboldtiana (Pulmonata). Gastropodia 2: 2-7, 11-12.

Webb, G. R. 1990. Notes on the sexology of Sonorella simmonsi Miller. Gastropodia 2: 22-24.

Webb, G. R. 1994a. Data on the sexology of four more Polygyra. Gastropodia 2: 36-38.

Webb, G. R. 1994b. The sexology of five species of Polygyra (Polygyridae Polygyrinae). Gastropodia 2: 30-31.

Wiswell, O. and H. Browning. 1967. Mating of the giant South American snail Strophocheilus oblongus. Anatomical Record 157: 409.

Woodruff, D. S. 1978. Natural history of Cerion 10: Evolution and adaptive radiation of Cerion-remarkably diverse group of West Indian land snails. Malacologia 17: 223-239.

Accepted: 9 March 2007 